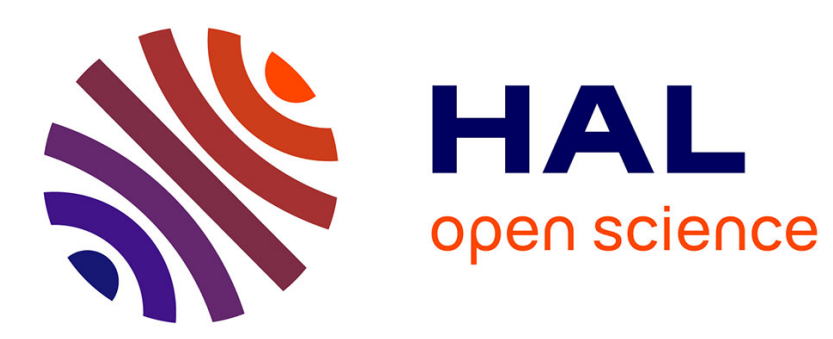

\title{
Droit, démocratie et liberté au travail dans le système français de relations professionnelles \\ Claude Didry
}

\section{To cite this version:}

Claude Didry. Droit, démocratie et liberté au travail dans le système français de relations professionnelles. Terrains et Travaux : Revue de Sciences Sociales, 2008, pp.à venir. hal-00210123

\section{HAL Id: hal-00210123 \\ https://hal.science/hal-00210123}

Submitted on 21 Jan 2008

HAL is a multi-disciplinary open access archive for the deposit and dissemination of scientific research documents, whether they are published or not. The documents may come from teaching and research institutions in France or abroad, or from public or private research centers.
L'archive ouverte pluridisciplinaire HAL, est destinée au dépôt et à la diffusion de documents scientifiques de niveau recherche, publiés ou non, émanant des établissements d'enseignement et de recherche français ou étrangers, des laboratoires publics ou privés. 


\title{
Droit, démocratie et liberté au travail dans le système français de relations professionnelles
}

Texte qui sera publié dans la revue Terrains et Travaux au mois de juin 2008

\author{
Claude Didry \\ didry@idhe.ens-cachan.fr
}

\section{Résumé :}

Le droit du travail n'est pas simplement le résultat d'un mouvement ouvrier constitué sur la base d'une organisation économique. Il se développe à partir de l'acquis révolutionnaire que représente le contrat de travail et conditionne la dynamique des luttes sociales. Il en résulte un processus qui traduit une causalité dialectique du droit sur les dynamiques économiques et sociales contredisant la vision naturaliste de l'économie et de l'histoire sociale classique.

\section{Introduction}

La dynamique des relations professionnelles se trouve associée, la plupart du temps, à celle d'un mieux-être social des salariés dont rend compte la notion d' " acquis social ». Dans cette perspective, le droit du travail est vu comme le produit dérivé d'un âge du « collectif» ${ }^{1}$ centré autour d'une "protection sociale» succédant au «désencastrement» libéral et industriel du XIXème siècle ${ }^{2}$. Soixante-dix ans après la victoire du Front Populaire, la globalisation et les nouvelles technologies sonneraient le glas d'un droit trop « rigide » pour les aspirations nouvelles des ingénieurs en informatique à la liberté sans frontière de l'Internet et pour la rigueur sur le coût du travail qui s'impose à des employeurs en proie à la tentation de la Chine. La fermeture des usines et la désindustrialisation accentueraient

\footnotetext{
${ }^{1}$. Cf. sur le collectif du siècle dernier porté par la « société salariale » et son infrastructure industrielle, cf. Castel (1993).

2. En suivant Polanyi (1983), le désencastrement correspond dans le monde du travail, à une définition du salariat comme contrainte de vendre sa force de travail pour gagner sa vie. Les syndicats sont des « facilitateurs» de ce processus en le rendant supportable.
} 
l'individualisation des rapports de travail. La désyndicalisation en France traduirait un effondrement du collectif d'antan et de la chaude solidarité ouvrière, auquel succéderait le froid individualisme de la France des propriétaires. Tout serait permis pour restaurer un droit qui prendrait l'eau de toute part, que les employeurs contourneraient sans difficulté avec, comme conséquence, des travailleurs perdant toute garantie sur leur emploi et leur salaire et sombrant, ainsi, dans la précarité ${ }^{3}$.

Dans une telle conception des relations professionnelles, comme univers relevant d'une prochaine histoire sociale, la démocratie n'aurait été qu'une valeur accidentelle, associée aux temps du collectif et de la fraternité. On concédera que les confréries ouvrières du XIXème siècle ne survivaient en France dans la clandestinité, que par le ciment d'un attachement à la République, dont l'insurrection des Canuts est une manifestation marquante (Sewell 1984). Mais il resterait ce péché originel de la Révolution, la loi Le Chapelier de 1791 enterrant les corporations avec le délit de coalition et la répression qui s'ensuivit. Par son individualisme, la Révolution aurait mis hors la loi la démocratie dans le travail.

Un retour sur la place du droit dans la dynamique du système français de relations professionnelles conduit cependant à s'interroger sur cette vision classique de l'histoire sociale. Avant toute grève, tout syndicat et toute mobilisation collective, c'est à partir de la référence au contrat de travail et au droit contractuel qui le définit, que le salarié envisage pratiquement sa condition. Ce constat ne devrait-il pas conduire à changer de regard sur le droit du travail et le droit dans le travail ? En réduisant le droit du travail aux seules réglementations limitatives de la liberté contractuelle, on oublie en effet que le contrat de travail lui-même est une catégorie juridique posée par le législateur, et donc l'Etat. Il existe donc une responsabilité publique à l'égard du contrat de travail, comme à l'égard de tout contrat, sur la base des principes constitutionnels posés par la Révolution. La Révolution conduit à l'affirmation de la liberté dans le travail, liberté individuelle comme maîtrise relative des individus sur leur avenir, mais aussi liberté collective comme influence plus ou moins organisée de la collectivité des travailleurs sur le devenir des activités économiques. Nous voudrions voir en quoi, sur la base de ce cadre juridique que constitue le contrat de travail, ce que l'on nomme le «mouvement ouvrier» correspond à une organisation de la démocratie dans le travail sur la base des valeurs de la Révolution.

\footnotetext{
${ }^{3}$. Le rapport Boissonnat (1995) a sonné l'hallali pour en finir avec un droit du travail usé et passer à un droit de l' « activité » dominé par la figure symbolique du «contrat d'activité » entre le travailleur et un groupement d'employeurs.
} 
Nous ne sommes pas des esclaves! le contrat entre liberté au travail et liberté du travail

Le contrat de travail, entendu de manière large comme la qualification contractuelle des rapports de travail ${ }^{4}$, implique de rapporter le travail à une référence juridique commune transcendant les professions. La «contractualisation» des rapports de travail qu'opère la Révolution implique ainsi l'existence d'un Etat centralisé, en mesure d'imposer sa loi aux groupes sociaux de toute nature : religieux, familiaux et professionnels. Elle s'appuie sur l'œuvre unificatrice du «droit révolutionnaire» (Weber 1986) rédigé de manière systématique à partir des valeurs du droit naturel formel (au centre desquelles se trouve la liberté de contracter).

Les rapports de travail s'inscrivent alors dans la grammaire du contrat telle que va la définir le Code civil après les grandes déclarations révolutionnaires. Cela conduit à analyser les rapports de travail comme une transaction portant sur l'échange salaire-emploi essentielle dans la définition du salariat (Waquet 1999). Le versement régulier d'une rémunération a ainsi pour contrepartie un emploi, c'est-à-dire l'exécution d'une activité définie par les stipulations contractuelles. Cette logique occultée du contrat conduit à limiter l'autorité de l'employeur à ce qui est nécessaire pour faire face aux aléas courants (Waquet op. cit.) . Le contrat de travail ne correspond donc pas à une forme d'esclavage à durée déterminée pendant laquelle le travailleur serait taillable et corvéable à merci, même dans les limites des bonnes mœurs. Il correspond à une référence que l'employeur et le travailleur pourront invoquer quand il leur semblera que l'autre partie va trop loin en regard des termes du contrat. Pour identifier l'influence causale méconnue de cette organisation juridique des rapports de travail, il faut revenir à la grande bifurcation historique que constitue la Révolution en France.

\section{La grève comme héritage méconnu de la Révolution}

La Révolution et le Code civil posent les bases d'un ancrage des rapports de travail dans le droit commun des contrats, qui conduisent à une mobilisation spécifique du Code civil devant les Conseils de prud'hommes. Comme le montre Cottereau (2002), l'identification d'une catégorie contractuelle adéquate pour qualifier les rapports de travail est précédée par une réflexion jurisprudentielle et doctrinale sur le « vrai louage ». Le « vrai louage » se définit

\footnotetext{
${ }^{4}$. En sortant de l'opposition entre les « contrats de louage » renvoyant à une forme de libéralisme anomique et le « contrat de travail » défini à partir de l'existence d'une base réglementaire plus substantielle à la fin du XIXème siècle avec les lois sur le temps de travail (1892) et les accidents du travail (1898).
} 
ainsi comme une transaction portant sur un travail défini, par opposition aux rapports de domesticité fondés sur la mise à disposition intégrale du travailleur au service de son patron et dénoncés alors comme une forme d' «esclavage ». Dans le monde du bâtiment parisien, le « vrai louage » correspond à un « travail à prix fait en place de grève ». Il s'oppose au travail de «journaliers » qui mettent leurs bras au service du patron. Le corrélat de ce contrat de louage est la liberté de quitter qui résulte de la suppression des corporations au lendemain de la nuit du 4 août 1789 avec l'abolition des privilèges, puis du décret d'Allarde et de la loi Le Chapelier de 1791. Cette liberté de quitter correspond à la remise en cause définitive du « délit de désertion » institué par les «Lettres Patentes pour entretenir la subordination parmi les ouvriers dans les pays manufacturiers » du 12 septembre $1781^{5}$. Elle ouvre aux ouvriers du bâtiment mécontents des conditions de leur travail, la possibilité de quitter leur patron pour retourner en Place de Grève. Au cours de la période qui précède l'adoption du Code civil, en 1804, le problème est ainsi, selon Cottereau, de limiter l'emprise de la domesticité et de garantir la liberté au travail des ouvriers hautement qualifiés qui se retrouvent sur la Place de Grève pour vendre leur travail à "prix fait". Ainsi, au lieu d'en tenir à une analyse exclusive du salariat comme une extension de la domesticité au travail ouvrier ${ }^{6}$, il faut envisager une causalité inverse et la permanence d'un monde du travail où les travailleurs ont une véritable marge de manœuvre sur la conduite de leur activité, comme le décrira, sous le Second Empire, Le Sublime de Denis Poulot.

\section{La fabrique collective comme système territorial de relations professionnelles}

Le contrat de louage comme transaction «à prix fait» met au centre de la scène sociale du XIXème siècle ceux que l'on nomme «façonnier» dans le textile ou encore « marchandeur » dans le bâtiment, voire, avec une connotation péjorative, «tâcheron ». Ces ouvriers prennent l'ouvrage que leur confient des négociants ou des entrepreneurs et se trouvent liés à leur donneur d'ouvrage par un contrat de louage d'ouvrage portant sur la livraison de pièces ou la réalisation de chantiers dont les tarifs sont fixés au préalable. Ils recrutent à leur tour des ouvriers qu'ils rémunèrent au temps. Les façonniers ${ }^{7}$ et les

\footnotetext{
5. «A la suite des réformes de Turgot, l'espoir d'émancipation avait au contraire été vivement déçu. Les anciennes règles de subordination, injonctions de soumission à la volonté des maîtres, poursuites pénales pour rupture de contrat ou manquements aux devoirs des inférieurs à l'égard des supérieurs, loin d'être abolies, avaient été rationalisées et unifiées nationalement (...)» (Cottereau 2002, p. 1536).

${ }^{6}$. Ce que suggèrerait les complexes industriels tels que Le Creusot en France.

${ }^{7}$. Par exemple les Canuts dans la soierie lyonnaise ou les rubaniers stéphanois
} 
marchandeurs ${ }^{8}$ deviennent les fers de lance dans la négociation de «tarifs » portant sur le prix des pièces ou des chantiers à réaliser ${ }^{9}$. Le tarif est le fruit d'une écriture collective, traduisant une forme de démocratie quasiment directe des travailleurs à façons. La mise en œuvre du tarif est revendiquée devant les tribunaux à titre d'usage de la profession ou dans le cadre de grèves, mais s'opère également la plupart du temps dans le cadre d'un contrôle informel passant par la surveillance de voisins susceptibles de prendre de l'ouvrage dans une période de pression à la baisse sur le prix de l'ouvrage (Reynaud 1992).

Ce schéma complexe, où l'on trouve d'une part un contrat liant le preneur d'ouvrage et le donneur d'ouvrage et d'autre part un contrat entre le preneur d'ouvrage et les ouvriers qu'il embauche ou trouve dans sa famille, est susceptible de s'appliquer dans de nombreux secteurs. On le retrouve dans les mines, avec la figure du haveur, immortalisée par le Maheu de Germinal qui emploie sa famille et éventuellement d'autres personnes (en l'occurrence E. Lantier) et qui prend l'ouvrage à travers la mise aux enchères des veines de charbon par la compagnie. On le retrouve dans des usines où le chef d'atelier (le «tâcheron ») est désigné comme l'employeur des ouvriers par le propriétaire des installations, en vue de décharger ce dernier de toute responsabilité en matière de contrôle des embauches et des conditions de travail $^{10}$.

\section{L'expérience de la conciliation et de l'arbitrage}

Cette dimension territoriale des rapports de travail et des grèves au XIXème siècle conduit à un rapprochement entre démocratie politique et démocratie professionnelle. Les ouvriers en grève se tournent vers les autorités locales, hommes politiques ${ }^{11}$ puis, avec la loi de 1892 sur la conciliation et l'arbitrage, juges de paix, pour organiser un débat entre les représentants des travailleurs et ceux du patronat. Les travailleurs désignent leurs représentants dans le cadre d'une pratique fondatrice pour la démocratie au travail: les assemblées générales. Le syndicat, tel que le définit la loi de 1884, est second. Il s'organise

\footnotetext{
${ }^{8}$. Tout en étant soumis à la dénonciation du «marchandage » (prohibé par le décret du 2 mars 1848) comme pratique de sous-traitance exonérant le donneur d'ordres de toute responsabilité dans le versement des salaires aux ouvriers engagés par le marchandeur en cas de faillite de ce dernier.

${ }^{9}$. Comme le tarif Colin de 1843 pour les marchandeurs du bâtiment parisien, mentionné dans Office du Travail (tome 1, 1899).

${ }^{10}$. Citons pour mémoire un jugement du tribunal de simple police de Saint-Didier La Séauve en Haute-Loire prononçant la relaxe d'un directeur d'usine «qui a soutenu que les contraventions ne pouvaient être retenues contre lui par le motif que les enfants mineurs, trouvés dans ses usines, n'étaient pas employés par lui, mais par des tâcherons, travaillant aux pièces, et payant eux-mêmes les enfants qu'ils occupent» (cité dans Didry et Brouté 2005), avant d'aboutir à un arrêt en cassation du 24 janvier 1902.
} 
fréquemment quand l'accord collectif de fin de grève ou le tarif est remis en cause et implique une action en justice contre le patron récalcitrant. La loi sur la convention collective du 25 mars 1919 porte la trace de cette prégnance de l'arbitrage et de la conciliation. La convention collective y est en effet définie comme « un contrat (...) entre les représentants d'un syndicat ou tout autre groupement d'employés et d'autre part les représentants d'un syndicat ou tout autre groupement d'employeurs, ou plusieurs employeurs contractant à titre personnel ou même un seul employeur. » Le « groupement » fait écho aux réflexions menées dans les rangs socialistes sur 1' " organisation de la grève » à partir de l'expérience de la conciliation et de l'arbitrage et avec notamment le grand projet de «conseils du travail» avancé en 1900 par Alexandre Millerand (Didry 2002). Il fait également écho aux commissions mixtes qui à un niveau régional (en fait surtout dans la région parisienne) ont arbitré les différends collectifs et déterminés les minima salariaux applicables aux industries d'armement.

Dans l'histoire du travail, la Révolution fixe le cadre juridique dans lequel se jouent aujourd'hui encore les rapports de travail. Ces rapports de travail se trouvent rapportés à un louage conçu comme transaction entre l'occupation d'un emploi et le versement régulier d'un salaire. La reconnaissance du droit de quitter qui résulte de la suppression des corporations consacre la liberté nouvelle des travailleurs, en leur donnant la possibilité de se retirer des situations de travail qui leur paraissent inacceptables. Elle oriente l'action ouvrière vers une pratique du retrait collectif, la grève, en bloquant la formation d'institutions plus durables appréhendées comme « coalitions » susceptibles de porter atteinte à un ordre libéral. Mais le libéralisme s'entend ici en deux sens au moins : ce que je propose de nommer la « liberté au travail » consiste à défendre le travail comme activité accomplie « en conscience » au regard des savoir-faire et des conditions de rémunération établis dans une profession. La liberté au travail dans sa dimension collective conduit ainsi, à travers la pratique de la grève, à la désignation de représentants en charge d'arriver à un accord permettant la reprise du travail. Elle se distingue de la liberté $d u$ travail comme liberté de «faire travailler» pendant une durée donnée, en réduisant le travail à un temps de subordination du salarié à l'employeur. Comme liberté de faire travailler, la liberté du travail émane du principe plus général de la liberté de l'industrie (Le Crom 2006).

\footnotetext{
${ }^{11}$. Comme le note M. Perrot (1974) pour les grèves des années 1880.
} 


\section{La dynamique institutionnelle du Front Populaire}

La rationalisation comme organisation de l'entreprise et lutte patronale pour le droit

$\mathrm{Au}$ lendemain de la Première Guerre mondiale, la rationalisation devient un des thèmes centraux de la politique économique en France (Fontaine 1925, Fridenson 1972). Elle devient un des leitmotivs d'instances nouvelles de démocratie sociale telles que le Conseil National Economique (Chatriot 2002), dans le cadre d'analyses qui demeurent macroéconomiques. Comme «motif idéologique» patronal (Moutet 1997), la rationalisation correspond cependant à un ensemble d'organisations du travail variant en fonction des activités économiques et de l'inspiration des employeurs. Elle désigne à la fois une pratique d'incitation salariale et la réorganisation du travail qui suit l'introduction de nouvelles machines. De manière générale, elle correspond à une redéfinition des conditions du travail conduisant, au niveau de l'entreprise, à une explicitation de l'emploi (par exemple sous la forme de la fiche de poste dans le cas du travail à la chaîne). La rationalisation correspond ainsi de manière plus profonde à un processus au terme duquel c'est l'entreprise elle-même qui prend une forme plus systématique, en intégrant les bureaux et les ateliers dans une chaîne hiérarchique allant de l'ouvrier au propriétaire du capital de l'entreprise, ce qui tranche avec la situation d'avant-guerre.

Cette dynamique large de rationalisation s'appuie sur le droit du travail qui s'est développé autour du contrat de louage puis de travail, avec l'adoption entre 1910 et 1919 d'un Code du travail qui agrège les différentes lois ouvrières. Le contrat de travail reste la base de référence des rapports de travail. Avec l'explicitation croissante des conditions d'emploi et les évolutions résultant de la mécanisation du travail, les employeurs tendent toutefois à affirmer leur autorité en poussant à la limite la marge d'aménagement des conditions du travail que leur reconnaît le droit contractuel pour faire face aux aléas productifs.

\section{Comité d'usine et convention collective}

La crise des années 1930 pousse les entreprises à aller plus loin dans le sens d'une affirmation de l'autorité patronale, avec une pratique fréquente de baisse des salaires, de violation et de dénonciation des conventions collectives et de licenciements. Dans le sillage de la pratique anarcho-syndicaliste des premières années du XXème siècle, la grève est vue comme une riposte à cette pression des employeurs, par la manifestation d'une certaine puissance ouvrière. Elle traduit la capacité d'entraînement des ouvriers syndiqués et non 
syndiqués, notamment dans les rangs de la CGT Unitaire proche du Parti Communiste, où la grève est vue comme la réalisation d'une « unité d'action à la base ». Cependant, à partir du début des années 1930, cette pratique commence à être remise en cause au vu de l'ampleur de la répression patronale. Benoît Frachon, le secrétaire général de la Fédération Unitaire des Métaux, membre (clandestin) du bureau politique du PCF souligne ainsi au cours du Comité central du Parti en octobre 1933 qu'en matière de grève, «Dans la région parisienne on a eu Citroën, mais après le mouvement, Citroën a développé son attaque, non sous une forme brutale, collective, comme il voulait le faire, mais dans chaque atelier et d'après les renseignements que nous avons, il en résulte dans certains cas que l'on a réalisé plus que ce dont on menaçait les ouvriers lors du mouvement. $\rangle^{12}$.

De manière moins visible que la réaction aux violences fascisantes du 6 février 1934 préfigurant le Front Populaire, la pratique des organisations syndicales évolue pour faire face aux problèmes les plus immédiats qui se posent dans les entreprises. Comme le montre Moutet (1997), les licenciements dans des entreprises textiles du Nord impliquent de la part des syndicalistes CGT et CGTU, une négociation sur l'évolution des conditions du travail, liée notamment à la mécanisation et aux gains de productivité qui en résultent. Dans les entreprises de la métallurgie, Badie (1972) note une inflexion de la stratégie dès 1933 dans le sens d'un travail d'écoute des « revendications immédiates des travailleurs ». La lutte des classes passe par une installation de militants ouvriers dans les entreprises, en vue de «mettre sur le papier » les revendications des travailleurs, à travers la pratique dès lors générale de la tenue de «cahiers de revendication». Cette pratique se cale sur l'institution que constitue, depuis la loi du 25 mars 1919, la convention collective.

En juin 1935, dans la dynamique générale de «Rassemblement Populaire », le Métallurgiste (organe de la Fédération Unitaire des Métaux) publie en une deux articles : à gauche un article de Benoît Frachon s'intitule «Imposons les contrats collectifs ! », à droite, un article d'Ambroise Croizat porte sur le «comité d'usine». Un lien explicite se trouve établi entre la convention collective et l'institution d'une représentation plus ou moins permanente des ouvriers. La convention collective devient ainsi la référence pour définir l'objectif de mobilisations collectives conçues comme des mouvements à moyen terme, partant d'une explicitation des revendications ouvrières relayées par des journaux d'entreprise (qui se nomment souvent l'Etincelle).

\footnotetext{
12. Archives du Parti Communiste Français, consultée par Claude Didry en mai 1999, extrait de Didry et Margairaz (2000).
} 
La grève de Saint-Chamond en novembre 1935 marque une étape décisive dans cette démarche nouvelle. Cette grève s'inscrit dans un ensemble de luttes contre la rationalisation menées, au cours de l'année 1935, dans les entreprises de la métallurgie de la Loire (Moutet 1997). A Saint-Chamond, la grève des Forges de la Marine est précédée par une analyse menée tout autant au sein des organisations syndicales unitaire que chrétienne. Comme le souligne Trimouille (1966) «Cette grève réussie semble, aux yeux des chrétiens, constituer un modèle: préparée par deux mois d'études préalables, déclenchée par un vote secret après épuisement des tentatives de conciliation dans une entreprise financièrement solide et pressée de livrer des travaux en cours (c'est une grève opportune), appuyée par les ouvriers unanimes derrière des dirigeants compétents, elle constitue "une magnifique leçon de syndicalisme professionnel"13 $»$ (Trimouille, 1966, p. 30). Dans le même esprit, un article intitulé «3.500 métallos de Saint-Chamond battent le Comité des Forges» est publié à la une du Métallurgiste de décembre 1935.

Le contrat collectif qui clôt la grève en décembre 1935 indique les grandes directions suivies dans la convention de la métallurgie de Saint-Étienne deux ans plus tard. Il traduit une remise en cause de l'organisation unilatérale de la hiérarchie en imposant une classification calée sur les trois catégories issues de la Guerre : ouvrier professionnel, ouvrier spécialisé et manœuvre. Il limite la portée des incitations salariales par une augmentation du salaire rémunérant la présence, «le salaire d'affûtage », et une diminution des primes. Enfin, il entérine l'élection de représentants de salariés, dans le prolongement du «comité d'usine» formé pendant la grève.

\section{La politique institutionnelle du Front Populaire}

Les grèves du Front Populaire ne sont pas le résultat d'une simple «explosion sociale » liée au mécontentement des travailleurs. La pratique liant la grève avec occupation d'usine, la négociation d'un accord collectif et l'élection de représentants des travailleurs a commencé à être rôdé dans certains cas exemplaires comme celui de Saint-Chamond. Cette pratique est préparée par un travail en amont d'écriture de cahiers de revendications relayées par des journaux d'usine. Elle s'appuie sur le cadre juridique de la convention collective et sur la désignation de représentants ouvriers telle qu'elle a été expérimentée dans les industries d'armement pendant la Première Guerre.

13. Expression tirée du Réveil social de la Loire, janvier 1936. 
La dynamique des grèves en mai-juin 1936 montre l'importance de ce travail préalable d'écriture des revendications ouvrières. En effet, c'est au sein des usines d'armement et d'aéronautique (en particulier Breguet au Havre le 12 mai) que le mouvement prend son essor. Ces grèves répondent aux licenciements de travailleurs pour fait de grève le $1^{\mathrm{er}}$ mai. Elles prennent cependant une orientation nouvelle en regard des succès électoraux du Front Populaire et du but que constitue la convention collective. Fort de cet objectif, les conflits durent et suscitent ainsi de nouvelles occupations dans les secteurs les plus divers. Les grandes usines ne rejoignent le mouvement que dans un second temps, du fait des difficultés d'implantation qu'y ont connues les militants ouvriers. Ainsi, contrairement à un économisme que l'on retrouve fréquemment dans l'histoire sociale classique, ce n'est pas le développement d'une industrie de masse (comme, par exemple, l'automobile) qui entraîne le reste de la population active. L'impulsion vient d'abord de secteurs moins liés à une production en série et plus ancrés dans des dynamiques d'innovation comme le montre la place des industries de l'armement et de l'aéronautique dans le mouvement (Didry et Salais 1995).

Dans un contexte marqué par la crise et la montée du chômage, ce n'est pas la voie d'une politique de l'emploi que privilégie le gouvernement de Front Populaire. Certes, la semaine de 40 heures répond tout autant à un souci immédiat de création d'emploi qu'à la volonté de changer la vie des salariés. Mais la reconnaissance d'un nouveau type de conventions collectives par la loi du 24 juin 1936, la convention susceptible d'être étendue par décret à l'ensemble des travailleurs concernés, vise d'abord à organiser la négociation collective en vue de restaurer l'autorité du contrat de travail menacée par l'autorité patronale. Les 100 premières conventions collectives étendues sont publiées au Journal Officiel en 1937 après que le Conseil National Economique eut exercé son contrôle sur la portée des textes (audelà de l'entreprise) et la représentativité des signataires. Loin de l'historicisme qui résulte de la focalisation sur $\mathrm{du}$ 《fordisme» comme système économique et social «total $»^{14}$, ces conventions étendues font apparaître une pluralité de secteurs ayant à répondre à des problèmes spécifiques.

Le modèle du « contrôle ouvrier » que l'on trouve en premier lieu dans la métallurgie parisienne et stéphanoise, tend ainsi à mettre l'accent, dans le texte même de la convention, sur la classification des travailleurs, avec le souci d'arriver à un classement des différentes activités professionnelles couvertes par la convention dans les trois grandes catégories 
d'ouvrier professionnel, d'ouvrier spécialisé et de manœuvres. L'objectif est ici de contrebalancer le pouvoir d'organisation patronal dans l'entreprise par une nomenclature reconnue au niveau de la branche, en imposant aux employeurs de faire la preuve que les salaires versés sont supérieurs aux minimums de branche et ainsi en traduisant les classifications d'entreprise dans les termes de la classification de branche.

Le modèle «productiviste» que l'on trouve dans de nombreuses conventions du secteur textile (notamment celle de «l'industrie cotonnière normande ») met l'accent sur la nécessité de maintenir un prix de revient faible. Il développe pour cela les moyens d'accroître la productivité du travail par des systèmes de primes venant s'ajouter au salaire en fonction de la production réalisée.

Enfin, dans un ensemble relativement hétérogène de secteurs marqués par un rapport au temps spécifique, les conventions collectives font une place importante à une organisation des horaires de travail qui permettront d'assurer la continuité du service (dans le cas de transports collectifs urbains) ou de faire face aux intempéries (dans le cas du bâtiment).

Par leur diversité, les conventions collectives de 1936 traduisent une dynamique institutionnelle ancrée dans des collectivités économiques singulières. La référence aux cadres juridiques permet ainsi un ancrage de la démocratie professionnelle dans les différentes activités économiques, sans qu'il ne soit possible de discerner l'influence causale d'ensemble d'une hypothétique structure économique telle que l'industrie fordiste, sur le droit du travail. La redécouverte du droit du travail comme ressource pour les travailleurs et leurs représentants constitue ainsi un des acquis essentiels du Front Populaire comme le souligne avec vigueur un des artisans du mouvement, le syndicaliste, député communiste (et futur ministre du travail), Ambroise Croizat :

«Il faut que chacun connaisse ses droits et agisse pour les faire respecter. Toute infraction aux lois sociales, toute violation des clauses du contrat ou des accords conclus doivent nécessiter l'intervention du ou des délégués. Si celui-ci ne suffit pas, il y a le syndicat et aussi les conseils de prud'hommes. Il sera, en outre, constitué dans chaque département des commissions paritaires qui auront pour mission d'examiner les conflits pouvant surgir et de rechercher une solution. » («La valeur des Lois sociales et des avantages acquis », L'Union des métaux, juin-juillet 1936).

${ }^{14}$. Comme modèle de « régulation économique » articulant production et consommation de masse (cf. Boyer 


\section{L'ancrage de la démocratie dans l'entreprise: l'articulation entre comités} d'entreprise et syndicats

Dans une période d'effervescence collective telle que le Front Populaire, la syndicalisation des salariés ne dépasse pas le tiers de la population salariée. La syndicalisation n'a donc jamais atteint en France les niveaux des pays scandinaves. Certes, la faiblesse dramatique de la syndicalisation ${ }^{15}$ aujourd'hui est lourde de menaces pour le devenir du droit du travail, les garanties des travailleurs et plus généralement la garantie d'un état de droit au travail. La dimension démocratique que porte en lui le travail salarié passe cependant en France par des voies spécifiques qui évoquent une forme de démocratie représentative où l'influence de l'acteur syndical ne se réduit pas au poids de ses effectifs. La pratique de la grève (telle qu'on a pu l'observer dans la grève récente de la SNCF) est à cet égard révélatrice. Loin d'apparaître comme une stratégie orchestrée par l'acteur syndical, elle est le fruit d'une décision collective prise dans le cadre d'assemblées générales où les représentants syndicaux viennent apporter des points de vue différents et susceptibles d'alimenter le débat. Cette dimension démocratique se manifeste également à travers un autre ensemble d'acteurs collectifs dont le rôle devient plus sensible dans le contexte de restructurations récurrentes : les comités d'entreprise. Les comités d'entreprise traduisent une capacité de délibération qui semble inégalée en Europe. Cette capacité s'affirme à travers l'influence de ces derniers dans les restructurations, tant pour limiter les suppressions d'emplois que pour orienter les recompositions des collectifs pertinents de travail.

Les instances représentatives du personnel comme maillage de la démocratie professionnelle

L'institution des comités d'entreprise par l'ordonnance du 22 février 1945 représente une forme d'aboutissement pour les projets visant à organiser la grève et, dans son prolongement, la représentation des salariés. Pour les entreprises de plus de cinquante salariés, les comités d'entreprise viennent alors s'ajouter aux délégués du personnel établis en 1936. Ces instances représentatives du personnel s'imposent alors que, dans le même temps, les commissions paritaires départementales ont disparu. Aujourd'hui, la représentation élective des salariés dans l'entreprise est assurée par au moins un délégué du personnel dans $72 \%$ des établissements de plus de 20 salariés (source : enquête REPONSE 2005, présentée dans Jacod 
2007). Si l'on y ajoute la présence de délégués syndicaux, il en résulte une représentation des salariés dans les entreprises de plus de 20 salariés atteignant $90 \%$ des travailleurs concernés. En sachant que la part des salariés dans les $\mathrm{TPE}^{16}$ est de $29 \%$, on arrive en gros à une couverture probablement supérieure au trois quarts de la population salariée par une IRP ${ }^{17}$.

Le poids des instances représentatives du personnel dans la population salariée permet de dégager une des plus fortes spécificités du système de relations professionnels français. En effet, si la syndicalisation est passée de $28 \%$ des salariés en 1950 à $8 \%$ des salariés en 2005 (Amossé et Pignoni 2006), le nombre d'instances représentatives du personnel s'est développé et la participation relativement forte aux élections aux comités d'entreprise (autour de 63\%) traduit le maintien d'une dimension collective dans les rapports de travail. Les syndicats tirent ainsi une bonne part de leur représentation de leurs résultats aux élections.

\section{Les élections aux comités d'entreprise ${ }^{18}$}

En 2005, la participation aux élections aux comités d'entreprise est de $63,2 \%$ des inscrits (salariés des entreprises de plus de 50 salariés). Elle diminue de 0,6 point par rapport à 2003. Les listes non syndicales recueillent 23,5\% des suffrages exprimés, contre 23,2\% en 2003. Elles obtiennent plus de la moitié des suffrages dans les établissements de moins de 100 salariés, alors que leur audience s'affaiblit dans les plus grosses unités au profit des listes syndicales. Avec 22,5\% des suffrages, la CGT redevient le premier syndicat les années impaires (où il n'y a pas d'élections à la $\mathrm{SNCF}$ ) pour la première fois depuis la fin des années quatre-vingt. La CFDT recueille 20,6 \% des suffrages exprimés et son audience baisse dans tous les collèges électoraux. Les syndicats non confédérés (UNSA, Solidaires...) progressent de 1,3 point entre 2003 et 2005 pour atteindre un score de 7,4\%. La CGT-FO, la CFTC et la CFE-CGC stabilisent leur audience avec respectivement $12,5 \%, 6,8 \%$ et $6,6 \%$ des suffrages exprimés.

Législation, démocratie et luttes sociales dans l'entreprise: le moment crucial du licenciement collectif

La diffusion d'une pratique démocratique dans l'entreprise -pour arriver à une influence véritable des représentants des salariés sur les décisions engageant l'avenir de l'entreprise- s'appuie sur l'existence d'un cadre légal organisant le fonctionnement des

\footnotetext{
15. Aux environs de $8 \%$ de la population salariée selon Amossé et Pignoni (2006).

16 . Très petites entreprises de moins de 20 salariés.

17. Sans tenir compte des délégués du personnel dans les TPE de 10 à 20 salariés, sachant qu'en 2005, 3,1 millions de salariés du secteur marchand non agricole sont employés dans des entreprises de moins de 10 salariés, soit en gros $12 \%$ de la population salariée.

${ }^{18}$. Source :
} 
instances représentatives du personnel et notamment celui des comités d'entreprise. Après les crispations idéologiques de la Guerre froide (Le Crom 2003), les lois dites Auroux de 1982 ont donné une impulsion nouvelle à la démocratie dans l'entreprise en introduisant la possibilité, pour le comité d'entreprise, de se faire assister par un expert de son choix rémunéré par l'employeur, dans le cadre d'une expertise annuelle des comptes de l'entreprise.

Les procédures d'information-consultation prévues en cas de licenciement collectif marquent un moment fort dans la mesure où elles touchent à l'avenir des contrats de travail. Ces procédures se sont étoffées depuis les premières restructurations des années 1960. La suppression de l'autorisation administrative de licenciement (requise pour les licenciement économique) puis le nouveau régime du licenciement économique, en 1986, ont permis de retrouver l' «antagonisme structurant» du contrat de travail, en éliminant le voile que représentait l'intervention administrative. Cette législation a ouvert aux instances représentatives du personnel la possibilité de saisir le juge judiciaire civil, notamment à partir de la loi du 27 janvier 1993 prévoyant la nullité de la procédure de licenciement collectif en cas de plan social (aujourd'hui plan de sauvegarde de l'emploi) non conforme aux dispositions légales.

Une analyse lexicale des décisions judiciaires publiées $^{19}$ que cette loi de 1993 a suscitées, nous a conduit identifier trois « registres d'action» des comités d'entreprise face aux licenciements collectifs : le conflit ouvert sur les compétences du comité d'entreprise et le respect de la procédure (registre $1^{20}$ ), la focalisation des débats sur les mesures d'accompagnement social des suppressions d'emploi, telles que les indemnités de départ et les propositions de reclassement (registre $2^{21}$ ). Enfin, par leur connaissance de l'activité liée au travail même des salariés, certains comités d'entreprise sont arrivés également à remettre en cause la bonne foi des arguments économiques avancés au soutien de la procédure de licenciement (registre $3^{22}$ ).

http://www.travail-solidarite.gouv.fr/etudes-recherche-statistiques/statistiques/relations-

professionnelles/institutions-representatives-du-personnel/comite-entreprise-

1950.html?var_recherche $=\%$ E9lections + au + comit $\% E 9+d \% 27$ entreprise, page consultée le 26/12/07.

${ }^{19}$. Elle est présentée dans article de 1998: «Les comités d'entreprise face aux licenciements collectifs, trois registres d'argumentation », Revue française de sociologie, ${ }^{\circ} 3$, sept, p. 495-534.

${ }^{20}$. Que j'ai nommé « résistance à la compétitivité ».

${ }^{21}$. Que j'ai nommé « approfondissement des politiques de l'emploi».

${ }^{22}$. Que j'ai nommé « critique économique». 
Vers une définition conjointe de l'entreprise

Après les conflits ouverts des années 1990, la pratique des comités d'entreprise connaît d'importantes évolutions. Depuis la loi du 3 janvier 2003, les « accords de méthode » traduisent une ouverture vers une négociation syndicale sur les modalités de l'informationconsultation du comité d'entreprise et sur le plan de sauvegarde de l'emploi (Jobert 2007). Cette négociation s'appuie sur la menace crédible d'une action en justice du comité d'entreprise susceptible de conduire à la nullité de la procédure de licenciement et prolonge ainsi la jurisprudence des années 1990.

Toutefois, dans le contexte de restructurations résultant de la multiplication des opérations de fusion-acquisition, un enjeu nouveau se fait jour pour les élus du personnel : celui que constitue la définition du « collectif pertinent » des salariés pour la consultation, en jouant sur l'articulation des différents niveaux de représentation des salariés. Ainsi, le comité d'entreprise européen ${ }^{23}$ ou le comité central d'entreprise permet d'arriver à une vue d'ensemble des projets des directions, en sortant d'une discussion sur les contreparties à la perte d'emploi. De plus, face à la filialisation des entreprises, la revendication d'une « unité économique et sociale» place le comité d'entreprise comme la véritable incarnation du collectif économiquement pertinent face à des redéploiements souvent enfermés dans des logiques financières. C'est ainsi l'existence d'une unité économique et sociale qui a permis à Canal Plus d'échapper à la dissolution dans Vivendi au moment de la fusion de ce groupe avec Seagram (aboutissant à l'entité Vivendi Universal) en 2000 (Didry 2008). La mise en place d'Unités économiques et sociales tant au sein du Crédit Agricole qu'au sein de la Société Générale traduit également, dans le secteur bancaire, le souci des élus du personnel d'éviter un éloignement des activités financières et des activités de dépôts (Meixner 2008). L'enjeu est simultanément de préserver les ressources des instances représentatives du personnel tout en maintenant dans leur périmètre des salariés qui, autrement, leur auraient échappé. Il en résulte une façon sensiblement différente d'aborder les questions d'emplois.

\section{Conclusion}

La qualification contractuelle des rapports de travail suppose un cadre juridique permettant d'identifier une transaction portant non pas sur la mise en location de la personne

\footnotetext{
23. Regroupant des représentants des travailleurs des différents pays européens où l'entreprise est implantée, conformément à la législation résultant de la transposition de la directive de 1996 (sur cette institution nouvelle et sa pratique, cf. Béthoux 2004).
} 
du salarié, mais sur une prestation définie de ce dernier en contrepartie d'un salaire. C'est sur cette base que le droit du travail, comme organisation de la liberté au travail, s'est édifié. Le droit du travail est une branche du droit civil, dans la mesure où il établit les garanties permettant une qualification véritablement contractuelle des rapports de travail (Supiot 1993). Un regard historique sur le droit du travail conduit alors à mettre en évidence une dynamique spécifiquement institutionnelle. Le droit existant conditionne en effet à chaque époque la manière dont les acteurs -tant les employeurs que les salariés et leurs représentantsenvisagent leur situation. Les collectifs de la démocratie professionnelle se construisent sur la base de l'architecture que dessine un état de droit s'imposant dans les situations de travail.

Pour saisir cette dynamique, il faut dépasser une conception classique des relations professionnelles comme analyse de la rencontre entre des acteurs collectifs considérés comme donnés (les syndicats salariés et les employeurs) dans un contexte économique considéré comme exogène (Dunlop 1993 (1958)). Il est nécessaire de sortir également de la conception instrumentale du droit auquel nous ont habituée les politiques de l'emploi, réduisant le contrat de travail à un ensemble de mécanismes dont la portée incitative doit être renforcée par l'altération des garanties préservant le salarié d'une forme d'arbitraire.

Le droit du travail est la référence sur la base de laquelle les acteurs organisent leurs rapports de travail, en partant des stipulations des contrats individuels jusqu'à la réglementation du temps de travail et l'organisation d'instances représentatives du personnel. Partant de ce donné historique, il reste à évaluer les apports de ce droit du travail à un développement économique où l'innovation est le produit de la liberté collectivement garantie des salariés dans leur travail. Il reste également à mesurer l'importance de la responsabilité publique, au-delà des politiques de l'emploi, dans les rapports de travail comme territoire à part entière d'un état de droit issu des valeurs fondatrices de la Révolution française.

\section{Bibliographie}

Amossé Thomas et Pignoni Maria-Thérésa, 2006, «La transformation du paysage syndical français depuis 1945 », Données sociales, Paris, INSEE, p. 405-412.

Badie Bertrand, 1972, «Les grèves du Front Populaire aux usines Renault», Le mouvement social, n81, "Le monde de l'automobile", sd. Patrick Fridenson, p. 69-109.

Béthoux Elodie, 2004, «Les comités d'entreprise européens en quête de légitimité », Travail et Emploi, nº8, pp. 21- 35. 
Boissonnat , 1995, Le travail dans vingt ans, Commissariat Général du Plan, Rapport de la Commission présidée par Jean Boissonnat, Paris, Editions Odile Jacob.

Castel Robert, 1993, Les métamorphoses de la question sociale. Une chronique du salariat, Paris, Fayard.

Chatriot Alain, 2002, la démocratie sociale à la française. L'expérience du Conseil National Economique, 1924-1940, Paris, La Découverte.

Cottereau Alain, 2002, «Droit et bon droit, un droit des ouvriers instauré puis évincé par le droit du travail », Annales HSS, vol. 57, n6, novembre-décembre, p. 1521-1561.

Didry Claude, 2002, Naissance de la convention collective, débats juridiques et luttes sociales en France au début du XXème siècle, Paris, Editions de l'Ecole des Hautes Etudes en Sciences Sociales.

Didry Claude, 2008, « De la restructuration de l'entreprise à la restructuration des relations professionnelles: le cas de Canal Plus», L'homme et la société n¹63-164, à paraître.

Didry Claude et Meixner Marie, 2007, Démocratie économique et capacité des travailleurs, la mise en oeuvre de la directive européenne "Information-consultation 》 de 2002 dans cinq pays européens (Suède, Royaume-Uni, France, Belgique, Allemagne), rapport de synthèse pour la Commission Européenne.

Didry Claude et Salais Robert, 1995, « Troubles sur les produits d'Etat et écriture des conventions collectives du travail en 1936 », in Jacob, A. Vérin, H. (ed) L'inscription sociale du marché, Paris, L'Harmattan, p. 112-136.

Dunlop John T., 1993, Industrial Relations Systems, revised edition, Boston, Harvard Business School Press, (1 ${ }^{\text {ère }}$ éd 1958).

Fontaine Arthur, 1925, La Guerre et l'industrie française, Paris, Presses Universitaires de France (Publications de la dotation Carnegie pour la paix internationale).

Fridenson Patrick, 1972, Histoire des usines Renault. Naissance de la grande entreprise : 1899-1939, Paris, Seuil.

Jacod Olivier, 2007, «Les instances représentatives du personnel: davantage présentes, toujours actives, mais peu sollicitées par les salariés », Premières synthèses, février, $\mathrm{n}^{\circ} 05-1$.

Le Crom Jean-Pierre, 2003, L'introuvable démocratie salariale, le droit de la représentation du personnel dans l'entreprise (1890-2002), Paris, Syllepse. 
Le Crom Jean-Pierre, 2006, " La liberté du travail en droit français. Essai sur l'évolution d'une notion à usages multiples », Diritto Romano Attuale, 15/giugno 2006 : Libertà lavoro diritto, p. 139-162.

Meixner Marie, 2008, «Co-defining the workplace: a comparison of employee representation », British Journal of Industrial Relations, à paraître.

Moutet Aimée, 1997, Les logiques de l'entreprise, la rationalisation dans l'industrie française de l'entre-deux-guerres, Paris, Editions de l'EHESS.

Office du Travail, (1899-1904), Les associations professionnelles ouvrières, 4 tomes, Paris, Imprimerie Nationale.

Perrot Michèle, 1974, Les ouvriers en grève, 2 tomes, Paris-La Haye, Mouton.

Polanyi Karl, 1983, La grande transformation, aux origines politiques et économiques de notre temps, Paris, Gallimard.

Poulot Denis, 1980, Le Sublime ou le Travailleur tel qu'il est en 1870 et tel qu'il peut être, Paris, Maspéro (1872).

Reynaud Brigitte, 1992, "Entre famille et métier: les passementiers stéphanois", Entreprise et histoire, $\mathrm{n}^{\circ} 1,1992$, pp. 79-80.

Sewell William H., 1983, Gens de métier et révolutions. Langage du travail de l'Ancien Régime à 1848, Paris, Aubier Montaigne, 1983.

Trimouille , 1968, « Aux origines du syndicalisme moderne d'inspiration chrétienne : les syndicats chrétiens dans la métallurgie française de 1935 à 1939 », Le mouvement social, $\mathrm{n}^{\circ} 62$, p. $27-47$.

Waquet Philippe, 1999, «Le renouveau du contrat de travail», Revue de Jurisprudence Sociale, 5, pp. 383-394. 